\title{
Minimizing Manufacturing and Quality Costs in Multiresponse Optimization
}

\author{
Jose Luis Duarte Ribeiro, Dr. \\ (ribeiro@vortex.ufrgs.br) \\ Flavio S. Fogliatto, Ph.D. \\ Carla S. ten Caten, M.Sc. \\ Department of Industrial \& Transportation Engineering \\ Universidade Federal do Rio Grande do Sul \\ Praça Argentina, 9 / Sala LOPP - Porto Alegre - RS - Brazil
}

\begin{abstract}
Most industrial processes and products are evaluated by more than one quality characteristic. To select the best design and operating control factors it is necessary to take into account all measures of quality simultaneously, in what is known as multiresponse optimization. Determination of the best operational settings for products or processes is usually accomplished through single-objective function optimization routines. Here we propose a new objective function for multiresponse optimization, based on Taguchi's loss function. Our function innovates in taking into account manufacturing and quality-related costs in the optimization, in addition to the usual criteria of minimum response variance and distance-totarget, and maximum robustness. We also propose a simplified procedure for modeling the variance of responses from non-replicated factorial designs. The paper contains a case study from the rubber industry.
\end{abstract}




\section{Introduction}

In many experiments it is important to evaluate the same experimental unit with respect to more than one response. Such experiments are known as multiresponse experiments and are used in the development and improvement of industrial processes and products. Responses are measured from experimental units obtained by testing different combination of levels for the control factors. Ultimately, we want to determine the best control factors settings such that responses present some desired properties; for example, small distance-to-target and variance. Determining such settings is ordinarily done using single objective-function optimization routines. Thus, a central problem in the analysis of multiresponse experiments is finding a suitable objective function that combines several responses as well as relevant information related to them.

A number of authors have suggested utility functions that may be used as objective functions in the optimization of multiresponse experiments. Approaches in the literature may be arranged in three groups: (i) response surface methodology approaches, including the works of Lind et al. [1], Myers \& Carter [2], Biles [3], Myers et al. [4], Myers \& Montgomery [5], Del Castillo [6], and Kim \& Lin [7]; (ii) approaches based on the desirability function, including the works of Harrington [8], Derringer \& Suich [9], Chang \& Shivpuri [10], Del Castillo et al. [11], and Fogliatto et al. [12]; and (iii) approaches based on Taguchi's Robust Design theory, including the works of Khuri \& Conlon [13], León et al. [14], Logothetis \& Haigh [15], Tribus \& Szonyl [16], Yum \& Ko [17], Raiman \& Case [18], Elsayed \& Chen [19], Pignatiello [20], Ribeiro \& Elsayed [21], León [22], and Vinning [23].

Typical criteria in multiresponse optimization include: $(i)$ distance-to-target of response outcomes, (ii) response variance, (iii) sensitivity of the response to small variations in the 
settings of the control factors, and (iv) inaccuracy of prediction. All criteria are to be minimized in the optimization. Criterion (ii) is recommended in cases where the variance of responses can be described as a function of the experimental control factors. Criterion (iii) is useful when experiments are carried out in pilot plants or labs to be eventually scaled up. Criterion (iv) captures the variability in the responses adjusted for how well they can be predicted, given the experiment performed to estimate the parameters in the prediction model.

All optimization criteria above relate to responses and their desired properties. However, in several experimental setups a response close to target or with small variance may imply in unacceptable manufacturing costs. Such costs relate to raw-material, labor, energy, etc. Typical examples are mixture experiments, where high usage of a given ingredient may result in undesirable costs. Some utility functions, like those based on Taguchi, search for control factor settings that minimize costs due to poor quality. Our objective is to search for an optimum that results in both low quality and low manufacturing costs.

In this paper we extend the quadratic loss function presented in Ribeiro \& Elsayed [21] to include minimum manufacturing costs as an optimality criterion in multiresponse optimization. We also suggest a new assessment of the quality loss (or proportionality) coefficient in the loss function. The extended quadratic loss function is explored on a case from the rubber industry. We also suggest a simplified approach for modeling the variance of responses in nonreplicated factorial experiments. Variance modeling typically requires experiments with several replications, which is often economically infeasible in practice. Approaches in the literature use either information from replicates [24], [25], residuals from response models [26], [27], or mixed response models with noise generated through simulation [28], [29]. Our approach for modeling the variance of a response uses the residuals from the regression model for the response mean 
and does not require replications of experimental treatments. The approach suggested here may be viewed as a simplified version of the one in Box \& Meyer [30], although developed independently.

Finally, we present a five-step procedure for multiresponse optimization and apply it to the case study data. The proposed steps are: (i) problem identification; (ii) experiment planning and execution; (iii) individual modeling of response mean and variance; (iv) choice of utility function and optimization criteria; (v) optimization proper. Steps $(i)$ - (iii) cover early stages of experimentation and relate mostly to data gathering. Steps (iv) and (v) are directly related to multiresponse optimization.

In the case study we present here a rubber mixture is to be optimized. Control factors to be adjusted are process parameters and some ingredients in the mixture. Samples from experimental treatments are evaluated with respect to ten response variables. Treatments are not replicated. Optimization goals include: (i) minimization of costs due to poor quality, and (ii) minimization of manufacturing costs. Criteria considered in $(i)$ include response distance-totarget, variance, and sensitivity to fluctuations in the settings of the control factors. Criteria considered in (ii) include raw-material and energy costs.

The rest of this paper is organized as follows. In the next section we present the quadratic loss function extended to handle manufacturing costs; we also give the details of the proposed calculation of the proportionality coefficient in the loss function. The third section presents the approach for variance modeling in non-replicated experiments. The fourth section gives the procedure steps for multiresponse optimization, which are illustrated by the case study. A Conclusion closes the paper in the last section. 


\section{Quadratic loss function with quality and manufacturing costs}

Consider a multiresponse experiment where there are $J$ responses $(j=1, \ldots, J), I$ treatment combinations $(i=1, \ldots, I)$, and $K$ control factors $(k=1, \ldots, K)$. The vector $\mathbf{x}=\left[x_{1}, \ldots\right.$, $\left.x_{K}\right]^{\prime}$ gives the settings for the control factors; if these settings correspond to treatment $i$, then $\mathbf{x}=$ $\mathbf{x}_{i} . Y_{j}(\mathbf{x})$ denotes the outcome of the $j^{\text {th }}$ response at $\mathbf{x}$; when $\mathbf{x}=\mathbf{x}_{i}, Y_{j}(\mathbf{x})=Y_{j i}$. Response predictions obtained from regression models are denoted by $\hat{Y}_{j}(\mathbf{x})$, meaning the predicted value of response $j$ at $\mathbf{x}$; when $\mathbf{x}=\mathbf{x}_{i}, \hat{Y}_{j}(\mathbf{x})=\hat{Y}_{j i}$.

A multivariate quadratic loss function was proposed by Ribeiro \& Elsayed [21] and given by:

$$
\hat{Z}(\mathbf{x})=\sum_{j=1}^{J} w_{j}\left[\left(\hat{Y}_{j}(\mathbf{x})-T_{j}\right)^{2}+\hat{\sigma}_{j}^{2}(\mathbf{x})+\sum_{k=1}^{K} \sigma_{k}^{2}\left(\frac{\partial \hat{Y}_{j}(\mathbf{x})}{\partial X_{k}}\right)^{2}\right]
$$

where $\hat{Z}(\mathbf{x})$ denotes the predicted loss at $\mathbf{x}, w_{j}$ is the importance weight for response $j, \hat{Y}_{j}(\mathbf{x})$ is the predicted value of response $j$ at $\mathbf{x}, T_{j}$ is the target for response $j, \hat{\sigma}_{j}^{2}(\mathbf{x})$ is the predicted variance of response $j$ at $\mathbf{x}$, and $\sigma_{k}^{2}$ is the variance of control factor $k$, as observed in shop floor full scale operations.

The first two terms inside the brackets in equation (1) are obtained by taking the expected value of Taguchi's univariate loss function [31]; they account for distance-to-target and variance of response outcomes, respectively. The last term was obtained from Oh [32] and gives the sensitivity of responses to fluctuations in the settings of the control factors. The weight $w_{j}$ is given by: 


$$
w_{j}=\frac{R I_{j}}{\Delta E_{j}^{2}}
$$

where $R I_{j}$ denotes the relative importance of response $j$ and $\Delta E_{j}$ represents one half of the interval formed by the specification limits for response $j$. A procedure for determining $R I_{j}$ is given in the case study section.

Equation (1) provides dimensionless loss values proportional to the low quality in the product or process under study. In our method, loss values must be converted to dollar values. For that purpose, a proportionality constant $p$ must be determined. As originally proposed by Taguchi, $p$ gives the dollar value corresponding to one unit of loss, and is determined using expert opinion [Taguchi et al. (31)]. We now suggest a simplified procedure for assessing $p$.

The key to our procedure is establishing the market value of products under extreme conditions for the set of quality characteristics (QCs). The idea is intuitive: product quality varies according to how QCs comply to given specifications. A product with a QC beyond spec limits may be rated "class B" and given a lower market price. Analogously, a product with all QCs close to target may be rated "class A" and given a premium market price. By determining two such classes of a product and their respective market values, we are able to find a realistic measure of the proportionality constant $p$.

Consider, for example, a class A product rated according to readings $\left(\hat{y}_{1 A}, \hat{y}_{2 A}, \hat{y}_{3 A}\right)$ of three QCs $(j=1,2,3)$; from appropriate substitutions in equation (1), we obtain a loss value of $\hat{Z}_{A}$. A class B product is characterized by readings $\left(\hat{y}_{1 B}, \hat{y}_{2 B}, \hat{y}_{3 B}\right)$ of the same QCs, with a predicted loss value of $\hat{Z}_{B}$. Market prices are $\$ \mathrm{~A}$ and $\$ \mathrm{~B}$ for class $\mathrm{A}$ and class $\mathrm{B}$ products, respectively. The proportionality constant $p$ is then given by: 


$$
p=\frac{\Delta \$}{\Delta Z}=\frac{(\$ A-\$ B)}{\left(\hat{Z}_{A}-\hat{Z}_{B}\right)} .
$$

Upon determining $p$, the predicted loss $\hat{Z}(\mathbf{x})$ at a certain control factor setting $\mathbf{x}$ may be converted to dollar values as follows:

$$
\hat{C}_{Q}(\mathbf{x})=p \times \hat{Z}(\mathbf{x})
$$

where $C_{Q}$ represents the monetary cost of poor quality.

We now want to incorporate manufacturing costs in our search for the best control factors settings. Start by computing relevant manufacturing costs (e.g., raw material and energy costs) at each treatment combination. Next, build a regression model relating control factors and manufacturing costs. Coefficients may be found deterministically. The general form of the model is:

$$
C_{M}(\mathbf{x})=\mathbf{X} \beta+\mathbf{u}
$$

where $C_{M}(\mathbf{x})$ denotes manufacturing costs at $\mathbf{x}, \mathbf{X}$ is the $(I \times R)$ information matrix of regressors with $R$ indicating the number of regressors, including the intercept, in the model, $\beta$ is a $(R \times 1)$ vector of regression coefficients, and $\mathbf{u}$ is a $(I \times 1)$ vector of residuals (when $\beta$ is found deterministically, $\mathbf{u}=\mathbf{0})$. Predictions from equation (4) are denoted by $\hat{C}_{M}(\mathbf{x})$.

An extended multivariate loss function to include manufacturing and poor quality costs is obtained by adding equations (3) and (4); i.e.,

$$
\hat{C}(\mathbf{x})=\hat{C}_{Q}(\mathbf{x})+\hat{C}_{M}(\mathbf{x})
$$

where $\hat{C}(\mathbf{x})$ denotes the overall costs incurred by running a process or manufacturing a product 
under conditions given by $\mathbf{x}$. The objective function in equation (5) is always to be minimized.

\section{A simplified approach to variance modeling}

An overview of our approach to variance modeling is as follows. Consider a factorial experiment with factors at $l$ levels and no replicates. That is a very common situation, since replication of treatments is usually costly and frequently not performed in practice. Suppose we determine a regression model relating outcomes of a response $j$ to levels of the control factors; only significant terms are included in the model. Using predictions from the model, we determine residuals at each treatment (i.e., difference between predicted and actual outcomes of $j$ ). The residuals give a measure of the variance in the experiment that could not be explained by the regression model. We want to check if residuals within a given level of a control factor differ significantly from those at other levels; when that is the case, response variance, as given by the residuals, may be considered to be a function of that factor.

There are six steps for determining regression models for the variance of a response $j$ :

1. Determine a regression model relating outcomes of response $j$ to experimental control factors.

2. For a given control factor $k$ plot the residuals at extreme levels and inspect for noticeable differences; when that is the case, proceed to step 3; otherwise, no model for the variance of $j$ is likely to be attainable.

3. Compute the sample variances for the residuals at each extreme level of $k$; say $s_{k,(-1)}^{2}$ and $s_{k,(+1)}^{2}$. We want to test the null hypothesis $H_{o}$ : variances are the same at extreme levels of $k$. For that purpose, an $F$-test is performed, as follows [33]: 


$$
F=\frac{s_{k,(s)}^{2}}{s_{k,(b)}^{2}},
$$

for a pair of extreme levels $s$ and $b$ of $k(s \neq b)$, using the greater of the two variance ratios.

Whenever $F \geq F_{\alpha / 2}$, variances are not the same at the two levels (use $\alpha \leq 0.05$ ) and should be modeled as a function of control factor $k$. Repeat steps 2 and 3 for all $K$ control factors.

4. List control factors that have effect on the variance of response $j$ and their sample variances at extreme levels. Organize results in a table. An example, with $M$ control factors and extreme levels labeled (-1) and (+1) for low and high, respectively, is given in Table 1.

\begin{tabular}{|c|c|c|c|c|}
\hline \multicolumn{4}{|c|}{ Control factors } & \multirow[b]{2}{*}{ Sample variances } \\
\hline$k=1$ & $k=2$ & $\ldots$ & $k=M$ & \\
\hline-1 & 0 & $\cdots$ & 0 & $s_{1,(-1)}^{2}$ \\
\hline+1 & 0 & $\cdots$ & 0 & $s_{1,(+1)}^{2}$ \\
\hline 0 & -1 & $\cdots$ & 0 & $s_{2,(-1)}^{2}$ \\
\hline 0 & +1 & $\cdots$ & 0 & $s_{2,(+1)}^{2}$ \\
\hline$\vdots$ & $\vdots$ & $\ddots$ & $\vdots$ & $\vdots$ \\
\hline 0 & 0 & $\cdots$ & -1 & $s_{M,(-1)}^{2}$ \\
\hline 0 & 0 & $\cdots$ & +1 & $s_{M,(+1)}^{2}$ \\
\hline
\end{tabular}

Table 1. Sample variances at extreme control factor levels of response $j$.

5. Build regression models relating sample variances and extreme levels for the control factors; these are the models for the variance of response $j$.

6. Repeat steps $1 \rightarrow 5$ for all remaining responses.

Some comments are noteworthy. The procedure above does not consider higher order terms (i.e., interactions and quadratic effects) when modeling the variance. That is precisely the reason for only checking on the sample variances at extreme levels. The benefits of considering higher order terms are dubious, for a number of reasons: (i) they are not likely to be significant 
in practice; (ii) estimates of higher order terms require a large number of data points, which are not usually available from factorial designs; and (iii) considering higher order terms would require a more complex model fitting procedure, which is not our objective.

As previously mentioned, the approach above is similar to the one in Box \& Meyer [30] although developed independently. There are two main differences: (i) Box \& Meyer provide means to incorporate interactions in the variance models; and (ii) determination of significant terms to be included in models is performed visually, through probability plots. Also, their approach is restricted to $2^{k}$ factorial designs.

\section{Steps for multiresponse optimization - a case study}

There are five steps to optimizing multiresponse experiments; they are: $(i)$ problem identification; (ii) experiment planning and execution; (iii) modeling of response mean and variance; (iv) choice of utility function and optimization criteria; (v) optimization proper. Those steps are briefly introduced in the sections that follow, and illustrated using case study data.

The case study involves a rubber product used as raw-material in the manufacturing of automobile tires. The product is manufactured at a chemical plant located in Brazil. The current formulation is based on specifications defined by clients. The experiment includes twenty-seven treatments consisting of product made with different formulations and under different processing conditions. Some of the ingredients are sulfur, synthetic rubber, and carbon black. Processing conditions relate to the total mixing time. The treatments are evaluated with respect to ten response variables; some responses are density, rheology measures (such as t10, t90 and MH), and hardness. Treatments are also evaluated regarding manufacturing costs. The goal is to find 
settings for the control factors that yield a low cost product with QCs closest to target, with minimum variance and sensitivity. In what follows, control factors and response variables are not identified to preserve confidentiality.

\section{A. Problem Identification}

Problem identification comprises information gathering on relevant response variables and control factors. In practice, listing of responses and control factors is not a complex task. However, choosing responses that reflect QCs relevant to customers and control factors that are most likely to affect responses is not trivial; that requires a group of individuals knowledgeable about the product or process under study.

We propose a scheme for prioritizing responses and control factors based on Quality Function Deployment principles [34, 35]; that is summarized in Figure 1. There are two matrices in Figure 1. Matrix A relates QCs and response variables; matrix B relates response variables and control factors.

There are three main inputs in matrix A: $(i)$ a list of $P$ QCs $(p=1, \ldots, P) ;(i i)$ importance scores $e_{p}$ for items in $(i)$; and (iii) a list of $J$ potential response variables $(j=1, \ldots, J)$ to be measured in the experiment. The list in $(i)$ should reflect customer's expectations about the product or process, and may be developed from joint efforts of a multifunctional team. Importance scores in (ii) are based mostly on expert opinion; decision analysis methods such as Saaty’s Analytic Hierarchy Process [36] may be helpful in establishing these scores. Items in (iii) correspond to measurable variables likely to describe QCs in (i); they are determined from technical considerations. Elements in (i) and (ii) are written in the rows of $\mathbf{A}$; elements in (iii) are written in the columns of $\mathbf{A}$. 


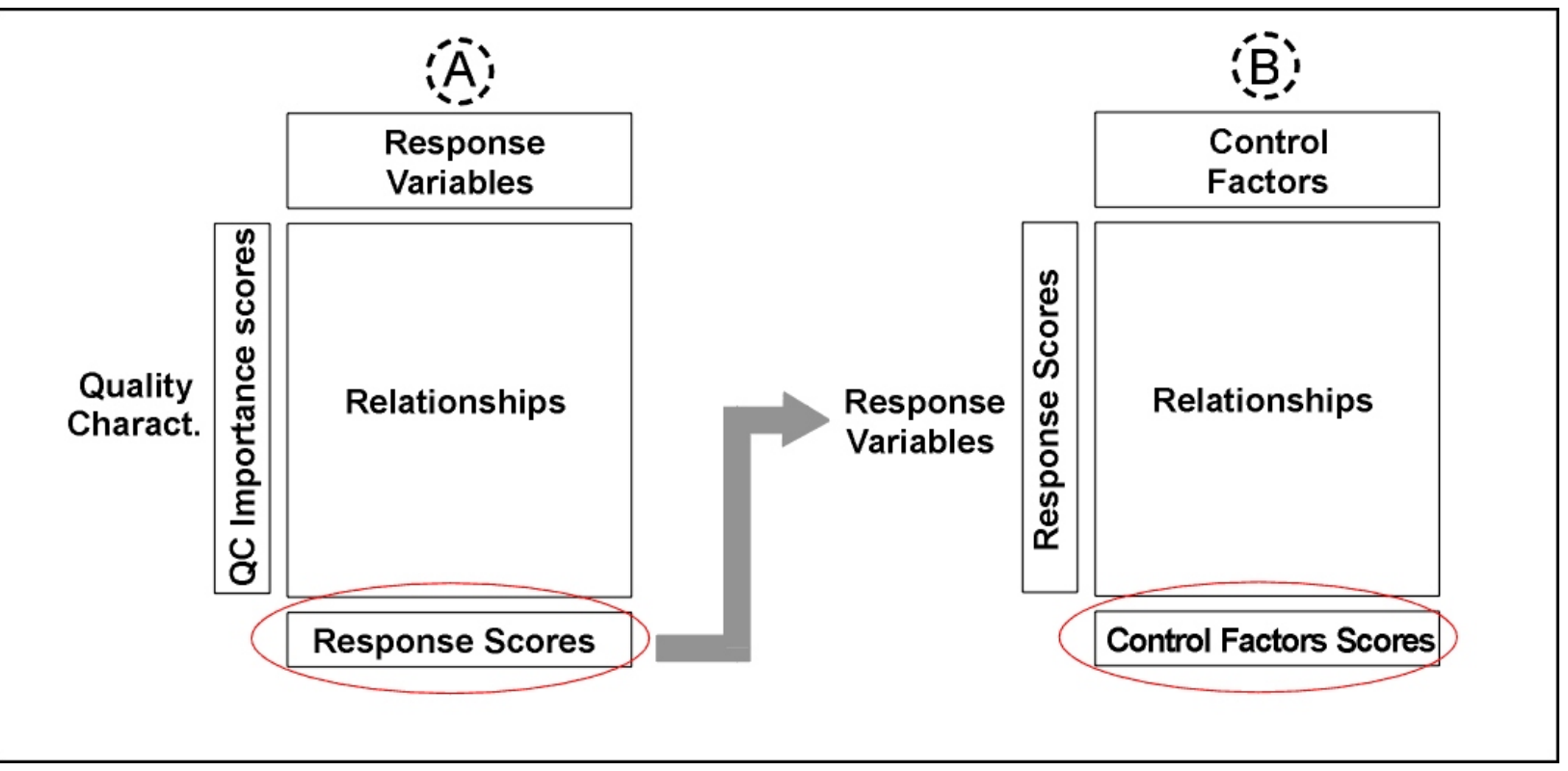

Figure 1. Matrices relating quality characteristics, responses, and control factors.

Each cell $(p, j)$ in matrix A gives a quantitative measure $a_{p j}$ of the relationship between row element $p$ and column element $j$. Evaluations are based on expert opinion and use a continuous scale with values from 0 (no relationship) to 9 (very strong relationship). An importance ranking for response variables may be obtained from scores given by:

$$
e_{j}=\sum_{p=1}^{P} e_{p} \times a_{p j} \text {, for all } j .
$$

Scores in equation (6) give the relative importance (RI) of responses, to be used when determining $w_{j}$ in equation (1).

Inputs in matrix $\mathbf{B}$ are: (i) a list of high scored response variables from matrix $\mathbf{A}$; (ii) importance scores for the response variables in (i); and (iii) a list of potential control factors to be explored in the experiment. Items in (iii) are determined based on technical considerations. Relationships in $\mathbf{B}$ are determined following the same reasoning presented for matrix A. An importance ranking for control factors may be determined based on scores obtained from 
equation (6) and the information in matrix $\mathbf{B}$.

The choice of responses and control factors to be considered in the experiment is based on their importance scores, as given in matrices $\mathbf{A}$ and $\mathbf{B}$ above. For each selected response list: (a) relative importance; (b) type (larger-is-best, smaller-is-best or nominal-is-best); (c) current observed value (if any); (d) target value; and (e) specification limits. For each selected control factor list: (a) current setting (if any); (b) allowable setting interval; and (c) flexibility (indicates difficulty in changing settings of a control factor).

Table 2 gives the responses selected in the case study and related information. Relative importance values in that table where obtained from a matrix similar to matrix $\mathbf{A}$ in Figure 1 and normalized to a $[0,5]$-scale with values rounded up to the next integer. For smaller-is-best (larger-is-best) responses, targets correspond to threshold values from which any smaller (larger) values are equally desirable and correspond to a zero loss situation.

Table 3 gives the control factors selected in the case study and related information. Flexibility is measured in a $[0,10]$-scale, where 10 denotes a control factor easily adjustable.

\section{B. Experiment Planning and Execution}

This section explores technical aspects to be considered when choosing an experimental design. Since the actual choice of design depends on each particular experimental setup, we restrict ourselves to providing general guidelines. Three main criteria direct the choice of experimental design; they are: (i) number of control factors; (ii) functional relationship between responses and control factors; and (iii) control factor flexibility.

A large number of control factors leads to experiments where many treatment combinations must be tested. For example, testing 5 factors at two levels each results in 32 
Minimizing Manufacturing and Quality Costs in Multiresponse Optimization

Jose L. D. Ribeiro (ribeiro@vortex.ufrgs.br), Flavio S. Fogliatto (ffogliatto@ppgep.ufrgs.br), arla S. ten Caten, (tencaten@vortex.ufrgs.br) Industrial Engineering \& Transportation Dept, Universidade Federal do Rio Grande do Sul, Praca Argentina, 9 / Sala LOPP, Porto Alegre, RS, Brazil. Phone: 5551 316-4005. FAX: 5551 316-4007.

treatment combinations. When large experiments are economically infeasible, fractional designs may be a good alternative. Thus, the number of control factors defines the degree of fractionating to be applied in a design. In fractional designs only a fraction of a replicate is run. In general, fractionating does not imply in significant loss of information; that is especially true as the number of control factors increases ([37], [38]). In any case, fractional designs can always be augmented when additional information on a particular factor is desired.

\begin{tabular}{|c|c|c|c|c|c|c|}
\hline $\begin{array}{c}\text { Response } \\
\text { Variable }\end{array}$ & $\begin{array}{c}\text { Relative } \\
\text { Importance }\end{array}$ & Type & $\begin{array}{c}\text { Current } \\
\text { Value }\end{array}$ & $\begin{array}{c}\text { Target } \\
\text { Value }\end{array}$ & \multicolumn{2}{c|}{$\begin{array}{c}\text { Spec Limits } \\
\text { Lower }\end{array}$} \\
\hline $\mathrm{Y}_{1}$ & 2 & Nominal & 8.0 & 8.5 & 7.93 & 9.07 \\
\hline $\mathrm{Y}_{2}$ & 2 & Nominal & 85 & 85 & 74.2 & 95.8 \\
\hline $\mathrm{Y}_{3}$ & 3 & Smaller & 220 & 210 & - & 232.7 \\
\hline $\mathrm{Y}_{4}$ & 3 & Nominal & 27 & 30 & 27.02 & 32.98 \\
\hline $\mathrm{Y}_{5}$ & 4 & Nominal & 62 & 62 & 59.49 & 64.51 \\
\hline $\mathrm{Y}_{6}$ & 4 & Nominal & 1.137 & 1.13 & 1.125 & 1.135 \\
\hline $\mathrm{Y}_{7}$ & 5 & Smaller & 80 & 65 & - & 78 \\
\hline $\mathrm{Y}_{8}$ & 4 & Larger & 1300 & 1400 & 1231.69 & - \\
\hline $\mathrm{Y}_{9}$ & 3 & Larger & 1900 & 2400 & 2328,02 & - \\
\hline$Y_{10}$ & 4 & Larger & 500 & 530 & 496.42 & - \\
\hline
\end{tabular}

Table 2. Responses and related information in the case study.

\begin{tabular}{|c|c|c|c|c|}
\hline $\begin{array}{c}\text { Control } \\
\text { Factor }\end{array}$ & \multirow{2}{*}{$\begin{array}{c}\text { Current } \\
\text { Setting }\end{array}$} & \multicolumn{2}{|c|}{$\begin{array}{c}\text { Allowable Setting Interval } \\
\text { Min }\end{array}$} & Flexibility \\
\hline $\mathrm{X}_{1}$ & 4.0 & 3.0 & 5.0 & 10 \\
\hline $\mathrm{X}_{2}$ & 6.5 & 5.5 & 6.5 & 10 \\
\hline $\mathrm{X}_{3}$ & 19.5 & 18 & 22 & 10 \\
\hline $\mathrm{X}_{4}$ & 3.5 & 2.0 & 4.0 & 10 \\
\hline $\mathrm{X}_{5}$ & 8 & 8 & 12 & 10 \\
\hline
\end{tabular}

Table 3. Control factors and related information in the case study.

Expected functional relationships between responses and control factors determine the number of levels at which control factors should be explored. Linear relationships are well explained by two-level designs; nonlinear relationships, however, require three or more levels for proper modeling. An efficient strategy is to start experimentation using a two-level design added of central points to check on nonlinear effects: in case they are significant, the design is 
Minimizing Manufacturing and Quality Costs in Multiresponse Optimization

Jose L. D. Ribeiro (ribeiro@vortex.ufrgs.br), Flavio S. Fogliatto (ffogliatto@ppgep.ufrgs.br), arla S. ten Caten, (tencaten@vortex.ufrgs.br) Industrial Engineering \& Transportation Dept, Universidade Federal do Rio Grande do Sul, Praca Argentina, 9 / Sala LOPP, Porto Alegre, RS, Brazil. Phone: 5551 316-4005. FAX: 5551 316-4007.

expanded to contemplate three levels of each control factor; for details, see Myers \&

Montgomery [5].

Control factor flexibility determines the degree of restriction on randomization. Flexible control factors are easily adjustable, with levels that may be completely randomized when running the experiment. Inflexible control factors are difficult to adjust and will be better held in blocked or split-plot designs. Consider the importance scores for the control factors determined from the approach in Figure 1. A low-scored inflexible control factor may be efficiently explored if confounded with blocks in a blocked design. A high-scored inflexible control factor will be better treated in a split-plot design; for details, see Hicks [38].

In Table 4, we suggest some commonly used designs that comply with the criteria above. In that table, CCD refer to Central Composite Designs [39]. Also, fractional CCD refer to CCDs in which the factorial portion is partitioned. In experiments where nonlinear effects are expected, a number of factors greater than two may be considered large; when expecting only linear effects, a number of factors greater than four may be considered large. For more information on suggested designs, see [37] and [38], among others.

\begin{tabular}{|c|c|c|c|}
\hline & & \multicolumn{2}{|c|}{ Number of control factors } \\
\hline & & Small & Large \\
\hline \multirow{2}{*}{$\begin{array}{c}\text { High } \\
\text { Flexibility }\end{array}$} & Linear & $2^{k}$ factorial & $\begin{array}{l}2^{k} \text { fractional factorial } \\
\text { Plackett-Burman }\end{array}$ \\
\hline & Nonlinear & $\begin{array}{c}3^{k} \text { factorial } \\
\text { CCD }\end{array}$ & $\begin{array}{c}3^{k} \text { fractional factorial } \\
\text { Fractional CCD }\end{array}$ \\
\hline \multirow{2}{*}{$\begin{array}{c}\text { Low } \\
\text { Flexibility }\end{array}$} & Linear & \multirow{2}{*}{\multicolumn{2}{|c|}{$\begin{array}{l}\text { Same as above. } \\
\text { Use blocking when dealing with low-scored control factors. } \\
\text { Use a split-plot strategy when dealing with high-scored control } \\
\text { factors. }\end{array}$}} \\
\hline & Nonlinear & & \\
\hline
\end{tabular}

Table 4. Recommended experimental designs according to selected criteria.

Table 5 gives the design matrix used in the rubber experiment. Given the large number 
of factors, high control factor flexibility and possibility of nonlinear effects, a fractional CCD was chosen. The experiment was run in three blocks. The first two blocks (eighteen experimental runs) correspond to a $2^{5-1}$ fractional factorial with two center points added to check on nonlinear effects, which resulted significant. According to technicians knowledgeable about the product, only control factors $\mathrm{X}_{1}$ and $\mathrm{X}_{2}$ could present nonlinear effects. Runs 24 to 27 correspond to the star portion of a CCD with two factors $(\alpha=1)$; they were run in a third block with five additional center points (runs 19 to 23 ). Notice that factors $\mathrm{X}_{3}, \mathrm{X}_{4}$, and $\mathrm{X}_{5}$ were held at center values in the second block. Runs were randomized within each block.

\section{Modeling of response mean and variance}

We want to develop regression models for the mean and variance of responses, using an appropriate modeling technique. There are four regression techniques available in the literature, each related to a different set of assumptions; they are: OLS (ordinary least squares regression), GLS (generalized least squares), MVR (multivariate regression), SURE (seemingly unrelated equations regression). OLS and GLS model responses individually; MVR and SURE model responses simultaneously. Choice of technique is driven by the degree of correlation among responses and homogeneity of responses variance. A summary of the assumptions for using each technique is given in Figure 2; these techniques are presented and compared in the context of multiresponse optimization in Fogliatto \& Albin [40].

Although not correlated among themselves, all but one of the responses in Table 2 presented non-homogeneous variances. Thus, GLS regression was used for modeling the means of responses, with weights given by the reciprocal of the predicted variances at each treatment. Resulting models are given in Table 6. Variance modeling followed the simplified approach 
Minimizing Manufacturing and Quality Costs in Multiresponse Optimization

Jose L. D. Ribeiro (ribeiro@vortex.ufrgs.br), Flavio S. Fogliatto (ffogliatto@ppgep.ufrgs.br), arla S. ten Caten, (tencaten@vortex.ufrgs.br) Industrial Engineering \& Transportation Dept, Universidade Federal do Rio Grande do Sul, Praca Argentina, 9 / Sala LOPP, Porto Alegre, RS,

Brazil. Phone: 5551 316-4005. FAX: 5551 316-4007.

previously presented; resulting models are also given in Table 6.

\begin{tabular}{|c|c|c|c|c|c|c||c|c|c|c|c|c|c|}
\hline Run & Block & $\mathbf{X 1}$ & $\mathbf{X 2}$ & $\mathbf{X 3}$ & $\mathbf{X 4}$ & $\mathbf{X 5}$ & Run & Block & $\mathbf{X 1}$ & $\mathbf{X 2}$ & $\mathbf{X 3}$ & $\mathbf{X 4}$ & $\mathbf{X 5}$ \\
\hline 1 & 1 & -1 & -1 & -1 & 1 & -1 & 15 & 2 & 1 & -1 & -1 & 1 & 1 \\
\hline 2 & 1 & -1 & -1 & -1 & -1 & 1 & 16 & 2 & 1 & 1 & 1 & -1 & -1 \\
\hline 3 & 1 & -1 & 1 & 1 & 1 & -1 & 17 & 2 & 1 & 1 & 1 & 1 & 1 \\
\hline 4 & 1 & -1 & 1 & 1 & -1 & 1 & 18 & 2 & 0 & 0 & 0 & 0 & 0 \\
\hline 5 & 1 & 1 & 1 & -1 & 1 & -1 & 19 & 3 & -1 & 0 & 0 & 0 & 0 \\
\hline 6 & 1 & 1 & 1 & -1 & -1 & 1 & 20 & 3 & 1 & 0 & 0 & 0 & 0 \\
\hline 7 & 1 & 1 & -1 & 1 & 1 & -1 & 21 & 3 & 0 & -1 & 0 & 0 & 0 \\
\hline 8 & 1 & 1 & -1 & 1 & -1 & 1 & 22 & 3 & 0 & 1 & 0 & 0 & 0 \\
\hline 9 & 1 & 0 & 0 & 0 & 0 & 0 & 23 & 3 & 0 & 0 & 0 & 0 & 0 \\
\hline 10 & 2 & -1 & 1 & -1 & -1 & -1 & 24 & 3 & 0 & 0 & 0 & 0 & 0 \\
\hline 11 & 2 & -1 & 1 & -1 & 1 & 1 & 25 & 3 & 0 & 0 & 0 & 0 & 0 \\
\hline 12 & 2 & -1 & -1 & 1 & -1 & -1 & 26 & 3 & 0 & 0 & 0 & 0 & 0 \\
\hline 13 & 2 & -1 & -1 & 1 & 1 & 1 & 27 & 3 & 0 & 0 & 0 & 0 & 0 \\
\hline 14 & 2 & 1 & -1 & -1 & -1 & -1 & & & & & & & \\
\hline
\end{tabular}

Table 5. Design matrix for the rubber experiment.

\begin{tabular}{|c|c|c|c|}
\hline \multirow{4}{*}{$\begin{array}{c}\text { Responses } \\
\text { variance }\end{array}$} & \multirow{4}{*}{$\begin{array}{c}\text { homogeneous } \\
\text { non- } \\
\text { homogeneous }\end{array}$} & \multicolumn{2}{|c|}{ Correlation among responses } \\
\hline & & correlated & uncorrelated \\
\hline & & MVR / SURE & OLS \\
\hline & & - & GLS \\
\hline
\end{tabular}

Figure 2. Summary of assumptions for using OLS, GLS, SURE and MVR regression.

Contour plots were obtained using the models for the mean of each response and some of the control factors. Figure 3 presents those plots for responses $Y_{1}$ to $Y_{4}$. It is clear from those plots that responses are closest to their targets at different control factors settings, demanding simultaneous optimization.

Recall that control factors correspond to process parameters and ingredients in a formula. Such ingredients in predefined quantities are processed in a special equipment, to give a desired rubber product. Process parameter and measurement variations are likely to occur when 
weighing and processing ingredients; these errors may be estimated to give a variability measure for the control factors to be used in equation (1). Variability associated with the control factors in the case study was measured from full scale in-plant operations. Estimates of $\sigma_{k}^{2}, k=1, \ldots, 5$ are given by:

$$
\hat{\sigma}_{1}=0.16 ; \hat{\sigma}_{2}=0.06 ; \hat{\sigma}_{3}=0.05 ; \hat{\sigma}_{4}=0.12 ; \hat{\sigma}_{5}=0.2 \text {. }
$$

\begin{tabular}{|l|l|l|}
\hline 1 & Mean & $\hat{Y}_{1}=7.10+1.08 X_{1}+0.64 X_{1}^{2}+1.11 X_{2}+0.54 X_{2}^{2}+0.42 X_{4}+0.256 X_{1} X_{2}(0.91)$ \\
\cline { 2 - 3 } & Var. & $\hat{\sigma}_{1}=0.362+0.186 X_{1}+0.118 X_{2}$ \\
\hline \multirow{2}{*}{2} & Mean & $\hat{Y}_{2}=74.62-2.33 X_{1}-6.26 X_{2}^{2}(0.39)$ \\
\cline { 2 - 3 } & Var. & $\hat{\sigma}_{2}=4.125-1.40 X_{3}+1.58 X_{5}$ \\
\hline \multirow{2}{*}{3} & Mean & $\hat{Y}_{3}=201.19-4.89 X_{1}-7.78 X_{1}^{2}-3.89 X_{2}-9.8 X_{5}(0.79)$ \\
\cline { 2 - 3 } & Var. & $\hat{\sigma}_{3}=6.225+2.525 X_{1}$ \\
\hline \multirow{2}{*}{4} & Mean & $\hat{Y}_{4}=31.57+3.60 X_{1}+1.43 X_{1}^{2}+1.98 X_{2}+1.58 X_{2}^{2}+1.69 X_{3}+1.10 X_{4}+2.36 X_{5}(0.93)$ \\
\cline { 2 - 3 } & Var. & $\hat{\sigma}_{4}=0.623+0.253 X_{2}$ \\
\hline \multirow{3}{*}{5} & Mean & $\hat{Y}_{5}=61.73+2.06 X_{1}+2.46 X_{1}^{2}+2.33 X_{2}+0.938 X_{3}+0.938 X_{5}(0.82)$ \\
\cline { 2 - 3 } & Var. & $\hat{\sigma}_{5}=1.633+0.892 X_{1}$ \\
\hline \multirow{3}{*}{6} & Mean & $\hat{Y}_{6}=1.132+0.00678 X_{1}+0.0058 X_{1}^{2}+0.0108 X_{2}+0.0063 X_{2}^{2}-0.00281 X_{4}(0.86)$ \\
\cline { 2 - 3 } & Var. & $\hat{\sigma}_{6}=0.00356+0.00202 X_{1}+0.00825 X_{4}$ \\
\hline \multirow{2}{*}{7} & Mean & $\hat{Y}_{7}=74.11-1.17 X_{1}-4.88 X_{4}+1.47 X_{5}+0.92 X_{1} X_{2}-0.689 X_{3} X_{4}(0.92)$ \\
\cline { 2 - 3 } & Var. & $\hat{\sigma}_{7}=0.5$ \\
\hline \hline \multirow{2}{*}{8} & Mean & $\hat{Y}_{8}=1602+335.56 X_{1}+179.47 X_{1}^{2}+228.67 X_{2}+154.47 X_{2}^{2}+167.75 X_{3}+125.75 X_{5}(0.91)$ \\
\cline { 2 - 3 } & Var. & $\hat{\sigma}_{8}=74.92+26.095 X_{2}$ \\
\hline \multirow{2}{*}{9} & Mean & $\hat{Y}_{9}=3306.4-76.24 X_{1}^{2}-123.44 X_{4}+41.19 X_{1} X_{2}(0.75)$ \\
\cline { 2 - 3 } & Var. & $\hat{\sigma}_{9}=53.03-23.56 X_{1}+18.52 X_{2}$ \\
\hline \multirow{2}{*}{10} & Mean & $\hat{Y}_{10}=520.7-58.1 X_{1}-32.6 X_{1}^{2}-34.2 X_{2}-22.6 X_{2}^{2}-32.7 X_{3}-12.1 X_{4}-21.6 X_{5}(0.93)$ \\
\cline { 2 - 3 } & Var. & $\hat{\sigma}_{10}=13.329-6.566 X_{2}-6.673 X_{3}$ \\
\hline
\end{tabular}

Table 6. Regression models for mean and variance of responses ( $R^{2}$ given in parentheses).

\section{Choice of Utility Function and Optimization Criteria}

A list of utility functions and criteria that may be considered when optimizing multiresponse experiments was given in the Introduction. Those utility functions consider 
different criteria and therefore may lead to different optima. Choice of utility function is essentially determined by the criteria to be considered in the optimization. Upon suitable adaptation, utility functions in the Introduction may handle any optimization criterion.

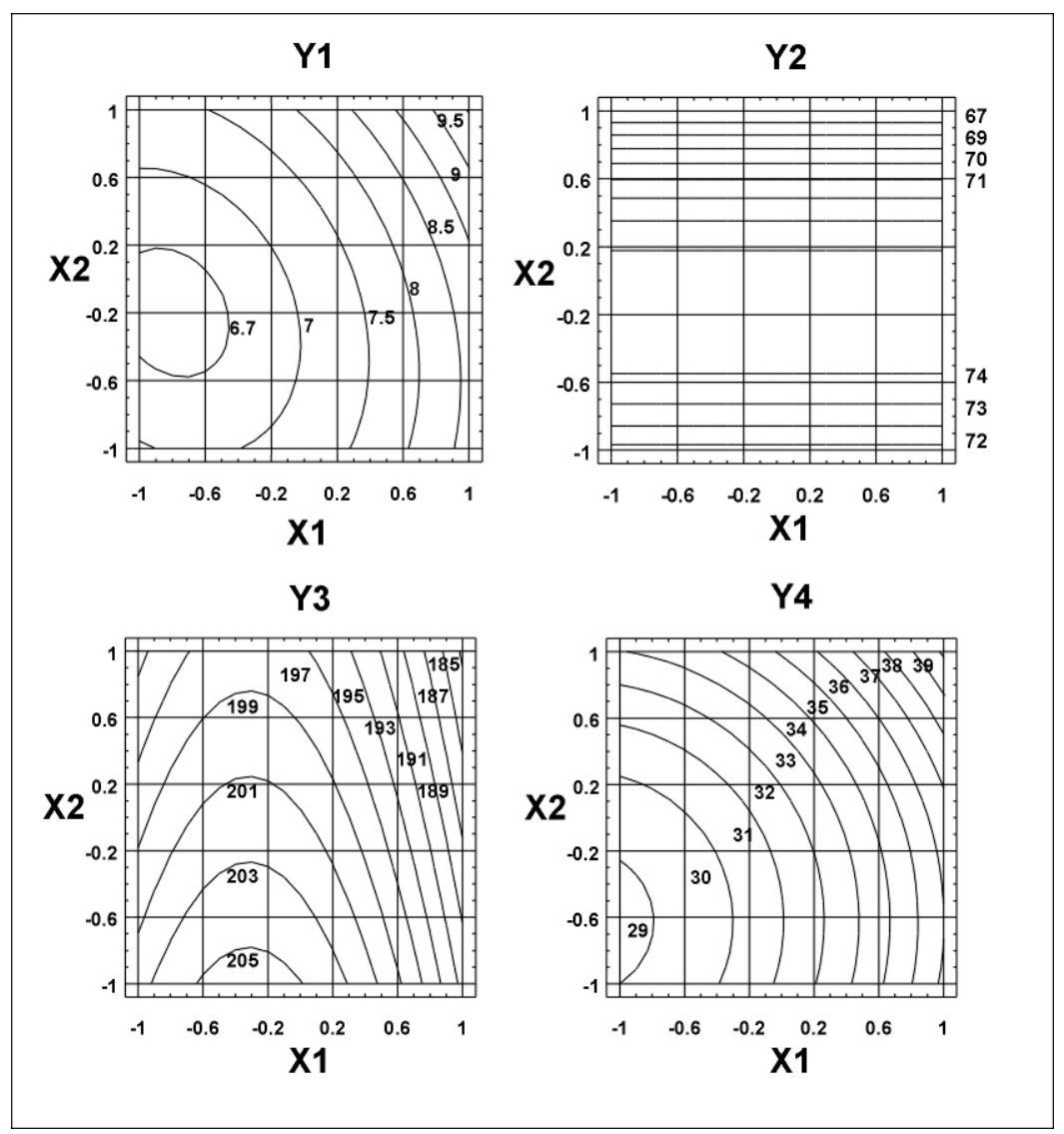

Figure 3. Contour plots for responses $Y_{1}$ to $Y_{4}$.

The utility function used to optimize the rubber experiment is the one suggested in equation (5), comprised of two terms: (i) a measure of monetary loss due to poor product quality, given in equation (3); and (ii) a measure of manufacturing costs given in equation (4). The proportionality constant $p$ to be used in equation (3), determined using our suggested procedure, is given by:

$$
p=\frac{\Delta \$}{\Delta Z}=\frac{(1.42-1.07)}{(179-64)}=0.003043
$$


Also, a model relating manufacturing costs and control factors, as proposed in equation (4), was determined to be:

$$
C_{M}(\mathbf{x})=1.42+0.0117 X_{1}-0.0156 X_{2}+0.00875 X_{4}+0.00375 X_{5} .
$$

In the case, manufacturing costs included raw-material and energy costs.

We now have all the information needed to perform the optimization using equation (5) as an objective function.

\section{E. Optimization Proper}

Upon determination of a suitable objective function (i.e., a utility function combining responses and relevant information related to them), nonlinear programming routines may be used to determine settings for the control factors where responses best satisfy the optimization criteria. Microsoft Excel [41] and Lindo's What's Best [42] are examples of computer packages able to perform nonlinear optimization.

Search for optimality in the rubber experiment was constrained by the design region (bounded by the upper and lower levels of the control factors); to conduct the search we used the Generalized Reduced Gradient (GRG) algorithm (Lasdon et al. [43]), a gradient-based optimization method available from Microsoft Excel [41] Solver option. Settings for the control factors that simultaneously optimize all responses and criteria are given in Table 7; they yield an expected cost of US\$ 1.598. Settings in Table 7 yield predicted outcomes for the responses in Table 8; notice that predicted outcomes at optimality are fairly close to target values.

To allow for a sensitivity analysis on optimization results, a regression model relating predicted costs at experimental treatments [as given by equation (5)] and control factors was 
Minimizing Manufacturing and Quality Costs in Multiresponse Optimization

Jose L. D. Ribeiro (ribeiro@vortex.ufrgs.br), Flavio S. Fogliatto (ffogliatto@ppgep.ufrgs.br), arla S. ten Caten, (tencaten@vortex.ufrgs.br) Industrial Engineering \& Transportation Dept, Universidade Federal do Rio Grande do Sul, Praca Argentina, 9 / Sala LOPP, Porto Alegre, RS, Brazil. Phone: 5551 316-4005. FAX: 55 51 316-4007.

determined. Figure 4(a) gives a contour plot obtained using that model and control factors $\mathrm{X}_{1}$ and $\mathrm{X}_{2}$; similar plots were obtained varying levels of different pairs of control factors. Figure 4(b) shows the effects on cost of varying the levels of control factor $\mathrm{X}_{1}$ (all remaining control factors are set at their optimal levels); Figure 4(c) gives similar results for control factor $\mathrm{X}_{2}$.

\begin{tabular}{|c|c|c|c|}
\hline Control Factors & Coded Levels & Control Factors & Coded Levels \\
\hline$X_{1}$ & -0.645 & $X_{4}$ & 1.00 \\
\hline$X_{2}$ & 0.475 & $X_{5}$ & -1.00 \\
\hline$X_{3}$ & 0.955 & \multicolumn{2}{l}{} \\
\hline
\end{tabular}

Table 7. Optimum settings for the control factors.

\begin{tabular}{|c|c|c|c|}
\hline Response & Specifications & Target Values & Predicted Value at Optimality \\
\hline$Y_{1}$ & $7.5-9.5$ & 8.5 & 7.66 \\
\hline$Y_{2}$ & $75-95$ & 85 & 72.10 \\
\hline$Y_{3}$ & $\leq 230$ & 210 & 209.14 \\
\hline$Y_{4}$ & $27-33$ & 30 & 31.49 \\
\hline$Y_{5}$ & $60-64$ & 62 & 62.49 \\
\hline$Y_{6}$ & $1.125-1.130$ & 1.13 & 1.134 \\
\hline$Y_{7}$ & $\leq 85$ & 65 & 67.57 \\
\hline$Y_{8}$ & $\geq 900$ & 1400 & 1638 \\
\hline$Y_{9}$ & $\geq 2300$ & 2400 & 3139 \\
\hline$Y_{10}$ & $\geq 500$ & 530 & 501.5 \\
\hline
\end{tabular}

Table $8 . \quad$ Predicted values of responses at optimality.

Inspecting contour plots and graphs like those in Figure 4 it was possible to determine a subset of the design region where control factors settings yield costs inferior to US\$1.70, which was considered acceptable by the manufacturer. Such subset is presented in Table 9, and is recommended for manufacturing the rubber product. Notice that control factor $\mathrm{X}_{1}$ presents the smallest interval of admissible settings; $\mathrm{X}_{1}$ corresponds to the most critical ingredient in the formulation.

\section{Conclusion}

This paper extends the quadratic loss function proposed in Ribeiro \& Elsayed [21] to 
include manufacturing costs as an optimality criterion in multiresponse optimization. We also suggest a simplified approach for modeling the variance of responses in non-replicated factorial experiments. Our approach uses residuals from the regression model for the response mean and may be viewed as a simplified version of the one in Box \& Meyer [30]. Finally, we propose a five-step procedure for multiresponse optimization based on the following steps: $(i)$ problem identification; (ii) experiment planning and execution; (iii) individual modeling of response mean and variance; (iv) choice of utility function and optimization criteria; $(v)$ optimization proper.

\begin{tabular}{|c|c|c|c|}
\hline Control Factors & \multicolumn{3}{|c|}{ Coded Levels } \\
\hline $\mathrm{X}_{1}$ & -0.80 & $\Leftrightarrow$ & -0.40 \\
\hline $\mathrm{X}_{2}$ & +0.20 & $\Leftrightarrow$ & +0.90 \\
\hline $\mathrm{X}_{3}$ & +0.60 & $\Leftrightarrow$ & +1.00 \\
\hline $\mathrm{X}_{4}$ & -0.40 & $\Leftrightarrow$ & +1.00 \\
\hline $\mathrm{X}_{5}$ & -1.00 & $\Leftrightarrow$ & +1.00 \\
\hline
\end{tabular}

Table 9. Subset of the design region yielding costs inferior to US\$1.70.

Developments proposed here are applied to a case study from the rubber industry. The product to be optimized is a rubber mixture. Five control factors are considered; they are process parameters and some key mixture ingredients. Samples from experimental treatments are evaluated with respect to ten response variables. Optimization goals are minimization of costs due to poor quality, and minimization of manufacturing costs. Criteria for optimality considered are response distance-to-target, variance, sensitivity to fluctuations in the settings of the control factors, and raw-material and energy costs. 


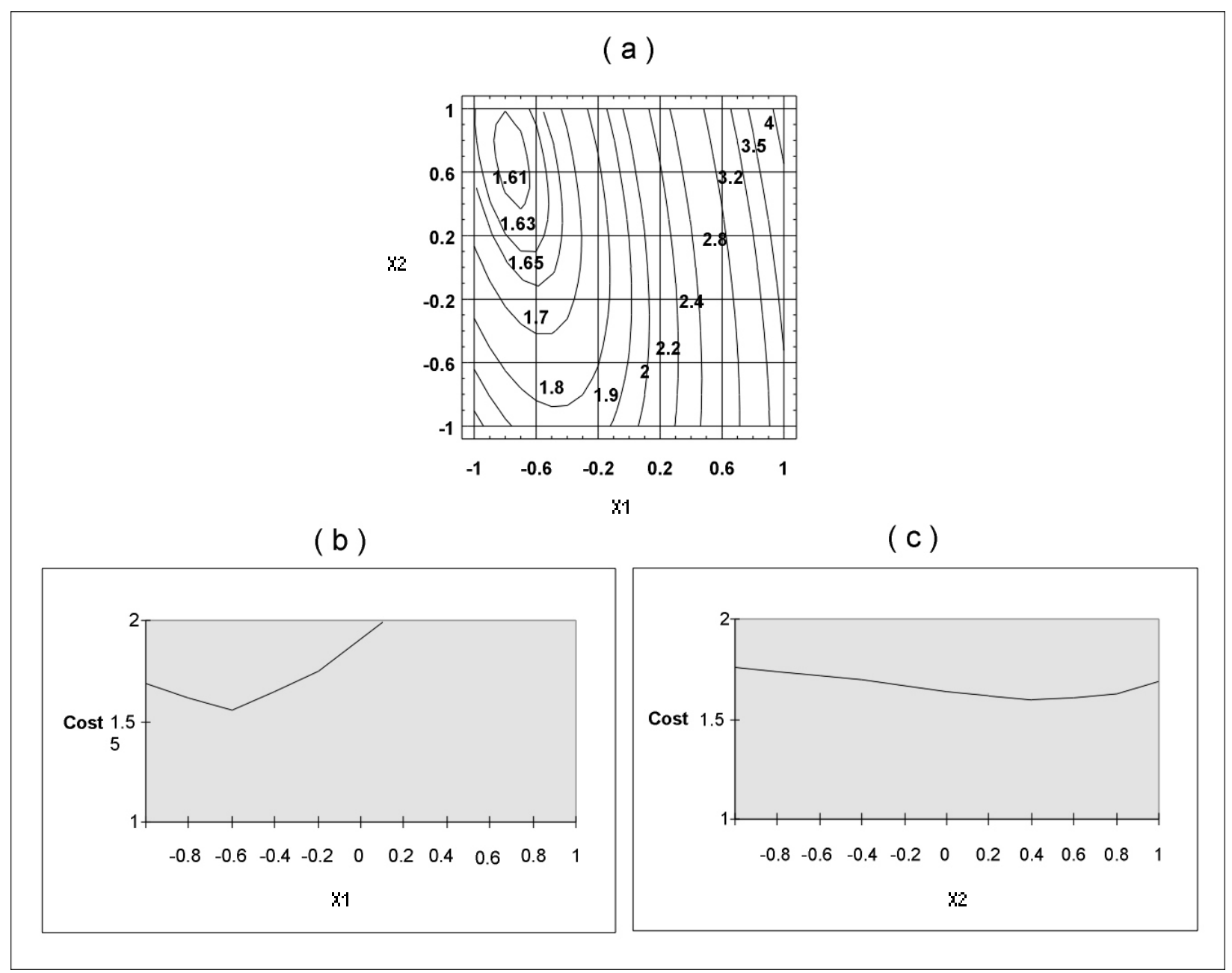

Figure 4. Sensitivity analysis on overall predicted costs as function of control factors $\mathrm{X}_{1}$ and $\mathrm{X}_{2}$.

\section{References}

[1] LIND, E. E., GOLDIN, J. \& HICKMAN, J. B. Fitting Yield and Cost Response Surfaces.

Chem. Eng. Prog., 56, 62-68, 1960.

[2] MYERS, R. H. \& CARTER, W. H. Response Surface Techniques for Dual Response

Surfaces. Technometrics, 15, 301-317, 1973.

[3] BILES, W. E. A Response Surface Method for Experimental Optimization of Multiresponse

Processes. Ind. Eng. Chemistry, Process Design and Development, 14, 152-158, 1975. 
[4] MYERS, R. H., KHURI, A. I. \& VINING, G. Response Surface Alternatives to the Taguchi Robust Parameter Design Approach. The American Statistician, 46 (2), 131-139, 1992.

[5] MYERS, R. H. \& MONTGOMERY, D. C. Response Surface Methodology. John Wiley, New York, 1995.

[6] Del CASTILHO, E. Multiresponse Process Optimization via Constrained Confidence Regions. J. Quality Technology, 28(1), 61-70, 1996.

[7] KIM, K. \& LIN, D. K. J. Dual response Surface optimization: A Fuzzy Modeling Approach. Journal of Quality Technology, 30 (1), 1-10, 1998.

[8] HARRINGTON, Jr., E. C. The Desirability Function. Ind. Quality Control, 21(10), 494498, 1965.

[9] DERRINGER, G. \& SUICH, R. Simultaneous Optimization of Several Response Variables. Journal of Quality Technology, 12(4), 214-219, 1980.

[10] CHANG, S. \& SHIVPURI, R. A Multiple-Objective Decision-Making Approach for Assessing Simultaneous Improvement in Die Life and Casting Quality in a Die Casting Process. Quality Engineering, 7, 371-83, 1994.

[11] Del CASTILHO, E., MONTGOMERY, D. C. \& McCARVILLE, D. R. Modified Desirability Functions for Multiple Response Optimization. J. Quality Technology, 28(3), 337-345, 1996.

[12] FOGLIATTO, F. S., ALBIN, S. L. \& TEPPER, B.J. A Hierarchical Approach to Optimizing Descriptive Analysis Multiresponse Experiments. Journal of Sensory Studies. Forthcoming, 1999. 
[13] KHURI, A. I. \& CONLON, M. Simultaneous Optimization of Multiple Responses

Represented by Polynomial Regression Functions. Technometrics, 23 (4), 363-375, 1981.

[14] LEÓN, R. V., SHOEMAKER, A. C. \& KACKER, R. N. Performance Measures Independent of Adjustment - An Explanation and Extension of Taguchi’s Signal-to-Noise Ratios. Technometrics, 29(3), 253-265, 1987.

[15] LOGOTHETIS, N. \& HAIGH, A. Characterizing and Optimizing Multiresponse Processes by the Taguchi Method. Quality and Reliability Eng. Int., 4, 159-169, 1988.

[16] TRIBUS, M. \& SZONYL, G. An Alternative View of the Taguchi Approach. Quality Progress, 22, 46-52, 1989.

[17] YUM, B. \& KO, S. On Parameter Design Optimization Procedures. Qual. Rel. Eng. Int., 7, 39-46, 1991.

[18] RAIMAN, L. B. \& CASE, K. E. The Development and Implementation of Multivariate Cost of Poor Quality Loss Function. IMSE Working Paper 92-152, Penn State Univ, 1992.

[19] ELSAYED, E. A. \& CHEN, A. Optimal Levels of Process Parameters for Products With Multiple Characteristics. Int. J. of Production Research, 31(5), 1117-1132, 1993.

[20] PIGNATIELLO Jr., J. J. Strategies for Robust Multiresponse Quality Engineering. IIE Transactions, 25, 5-15, 1993.

[21] RIBEIRO, J. L. \& ELSAYED, E. A. A Case Study on Process Optimization Using the Gradient Loss Function, Int. J. Prod. Res., 33 (12), 3233-3248, 1995.

[22] LEÓN, N. A. A Pragmatic Approach to Multiresponse Problems Using Loss Functions. Quality Engineering, 9, 213-220, 1996. 
[23] VINING, G.G. (1998). A Compromise Approach to Multiresponse Optimization. Journal of Quality Technology, 30 (4), 309-313.

[24] BARTLETT, M.S. \& KENDALL, D.J. (1946). The statistical analysis of varianceheterogeneity and the logarithmic transformation. Journal of the Royal Statistical Society, Ser. B, 8, 128-138.

[25] MYERS, R.H. \& MONTGOMERY, D.C. (1995). Response Surface Methodology: process and product optimization using designed experiments. John Wiley: New York.

[26] HARVEY, A.C. (1976). Estimating regression models with multiplicative heteroscedascity. Econometrica, 44 (3), 461-465.

[27] CARROLL, R.J. \& RUPPERT, D. (1988). Transformation and weighting in regression. Chapman \& Hall: New York.

[28] SHOEMAKER, A.C., TSUI, K.L. \& WU, C.F.J. (1991). Economical experimentation methods for robust design. Technometrics, 33 (4), 415-427.

[29] LUCAS, J.M. (1994). How to achieve a robust process using response surface methodology. Journal of Quality Technology, 26 (4), 248-260.

[30] BOX, G.E.P. \& MEYER, R.D. (1986). Dispersion effects from fractional designs. Technometrics, 28 (1), 19-27.

[31] TAGUCHI, G., ELSAYED, E.A. \& HSIANG, T.C. (1989). Quality Engineering in Production Systems. M $^{\mathrm{c} G r a w-H i l l: ~ N e w ~ Y o r k . ~}$

[32] OH, H.L. (1988). Variation Tolerant Design. Proc. Conf. Uncertainty in Eng. Des., Maryland. 
[33] FREUND, J.E. \& SIMON, G.A. (1997). Modern Elementary Statistics. $9^{\text {th }}$ Ed., Prentice Hall: Englewood Cliffs (NJ).

[34] AKAO, Y. (1990). Quality Function Deployment: Integrating Customer Requirements into Product Design. Productivity Press: Cambridge.

[35] COHEN, L. (1995). Quality Function Deployment: how to make QFD work for you. Addison-Wesley: Reading (MA).

[36] SAATY, T. L. (1980). The Analytic Hierarchy Process: Planning, priority setting, resource allocation. McGraw-Hill: New York.

[37] MONTGOMERY, D. C. (1991). Design and Analysis of Experiments. $3^{\text {rd }}$ Ed., John Wiley: New York.

[38] HICKS, C. R. (1993). Fundamental Concepts in the Design of Experiments. $4^{\text {th }}$ Ed., Saunders College Publishing: New York.

[39] BOX, G. E. P. \& DRAPER, N. R. (1987). Empirical Model-Building and Response Surfaces. John Wiley: New York.

[40] FOGLIATTO, F. S. \& ALBIN, S. L. (1999). Variance of Predicted Response as an Optimization Criterion in Multiresponse Experiments. Quality Engineering (forthcoming).

[41] MICROSOFT EXCEL, Ver. 5.0 (1994). User's Guide. Microsoft Co., Redmond, WA.

[42] WHAT'S BEST. (1998). User's Manual. Lindo Systems Inc., Chicago.

[43] LASDON, L.S., WAREN, A.D., JAIN, A.\& RATNER, M. (1978). Design and Testing of a Generalized Reduced Gradient Code for Nonlinear Programming. ACM Trans.

Math.Software,4,34-50. 
Key-Words: Multiresponse optimization, manufacturing costs, experimental design, rubber industry.

Jose Luis D. Ribeiro is Professor and Head of Department in the Industrial and Transportation Engineering Department at the Federal University of Rio Grande do Sul, Brazil. His research field is quality engineering. His work has been applied in areas including electronics manufacturing, rubber transformation and food processing. Dr. Ribeiro received his doctorate from the Federal University of Rio Grande do Sul in 1989. Dr. Ribeiro is serving as President of ABEPRO, the Brazilian Society of Industrial Engineers. He is a member of ASQ.

Flavio S. Fogliatto is Research Fellow in the Department of Industrial and Transportation Engineering at the Federal University of Rio Grande do Sul, Brazil. He received his Ph.D. in Industrial \& Systems Engineering from Rutgers University in 1997. His research field is quality engineering and stochastic modeling. His professional experience is in research and development of food products and processes. He is a member of ASQ.

Carla S. ten Caten is currently a Ph.D. student in the Department of Ceramics at the Federal University of Rio Grande do Sul, Brazil. She received her M.S. degree in industrial engineering from the Federal University of Rio Grande do Sul. Her research interests are in the area of statistical quality engineering. 